\title{
Dental Student Academic Integrity in U.S. Dental Schools: Current Status and Recommendations for Enhancement
}

\author{
Bruce S. Graham, DDS, MS, MEd; G. William Knight, DDS, MS, MS; \\ Linda Graham, BScN, MS
}

Abstract: Cheating incidents in 2006-07 led U.S. dental schools to heighten their efforts to enhance the environment of academic integrity in their institutions. The aims of this study were to document the measures being used by U.S. dental schools to discourage student cheating, determine the current incidence of reported cheating, and make recommendations for enhancing a culture of integrity in dental education. In late 2014-early 2015, an online survey was distributed to academic deans of all 61 accredited U.S. dental schools that had four classes of dental students enrolled; 50 (82\%) responded. Among measures used, $98 \%$ of respondents reported having policy statements regarding student academic integrity, $92 \%$ had an Honor Code, $96 \%$ provided student orientation to integrity policies, and most used proctoring of final exams (91\%) and tests (93\%). Regarding disciplinary processes, $27 \%$ reported their faculty members only rarely reported suspected cheating (though required in $76 \%$ of the schools), and $40 \%$ disseminated anonymous results of disciplinary hearings. A smaller number of schools $(\mathrm{n}=36)$ responded to the question about student cheating than to other questions; those results suggested that reported cheating had increased almost threefold since 1998. The authors recommend that schools add cheating case scenarios to professional ethics curricula; disseminate outcomes of cheating enforcement actions; have students sign a statement attesting to compliance with academic integrity policies at every testing activity; add curricular content on correct writing techniques to avoid plagiarism; require faculty to distribute retired test items; acquire examination-authoring software programs to enable faculty to generate new multiple-choice items and different versions of the same multiple-choice tests; avoid take-home exams when assessing independent student knowledge; and utilize student assessment methods directly relevant to clinical practice.

Dr. Graham is Professor of Restorative Dentistry and Dean Emeritus, College of Dentistry, University of Illinois at Chicago; Dr. Knight was Interim Dean, College of Dentistry, University of Illinois at Chicago, when this study was conducted and is now Senior Associate Dean of Academic Affairs, School of Dentistry, Oregon Health \& Science University; and Ms. Graham is Senior Research Specialist, College of Dentistry, University of Illinois at Chicago. Direct correspondence to Dr. Bruce S. Graham, Department of Restorative Dentistry (MC 555), University of Illinois at Chicago College of Dentistry, 801 South Paulina Street, Room 530D, Chicago, IL 60612-7212; 312-996-2669; bgraham@uic.edu.

Keywords: dental education, dental students, ethics, professional ethics, assessment, academic integrity, cheating

Submitted for publication 3/24/15; accepted 6/25/15

I n 2006-07, dental student cheating incidents at U.S. dental schools led to a Symposium on Integrity and Ethics in Dental Education convened by the American Dental Association (ADA) in June 2007. ${ }^{1}$ The U.S. and Canadian dental school deans subsequently studied the issue at their meeting in December 2007. Donald L. McCabe, a prominent researcher on the subject of college student cheating, addressed the deans' meeting. McCabe and other speakers at the conference recommended initiatives intended to reinforce a culture of academic integrity in dental schools.

In March 2005, Andrews et al. modified two survey instruments used by McCabe and administered them online to collect the anonymously reported perceptions of students and faculty about student academic integrity at U.S. and Canadian dental schools. ${ }^{2}$ In that study, 1,153 dental students responded to the survey. (As an indication of the response rate, there were 18,315 dental students enrolled in U.S. dental schools in 2004-05. ${ }^{3}$ ) Andrews et al. found that $74.7 \%$ of the respondents admitted cheating on written tests/ examinations and $68.4 \%$ admitted cheating on preclinical examinations/tests or assignments. In 1997, Beemsterboer et al. surveyed academic deans at 55 U.S. dental schools about student academic integrity. ${ }^{4}$ Of the 46 schools that responded (response rate of $84 \%$ ), 37 reported an average of 1.45 cheating cases per school per year during the 1996-97 time period. The aims of our study were to document the measures 
used by U.S. dental schools to discourage cheating, determine the current incidence of reported student cheating, and make recommendations for enhancing a culture of academic integrity in dental education.

\section{Methods}

This study, "Student Academic Integrity in U.S. Dental Schools, Protocol \#2014-0540," was approved by expedited review on July 28, 2014, by the University of Illinois at Chicago Office for the Protection of Research Subjects Institutional Review Board. During the period September 2014 through January 2015, emails were sent and some reminder telephone calls were made to the associate/assistant deans for academic affairs (or other relevant administrative title) at 61 accredited U.S. dental schools. Four other accredited U.S. dental schools were not included because they did not have four dental student classes enrolled at the time.

Table 1. Number of U.S. dental schools that were sent survey and responded, by type of school

\begin{tabular}{lcc} 
Type & $\begin{array}{c}\text { Number Sent } \\
\text { Survey }\end{array}$ & $\begin{array}{c}\text { Number That } \\
\text { Responded }\end{array}$ \\
\hline Public & 38 & 32 \\
Private, nonprofit & 20 & 15 \\
Private, state-related & 3 & 3 \\
Total & 61 & 50
\end{tabular}

Source of type of schools sent survey: American Dental Association. Survey of dental education: report 1, 2013-14. Table 1. At: www.ada.org/ /media/ADA/Science $\% 20$ and $\% 20$ Research/HPI/Files/SDE1_2013-14\%20_final.ashx. Accessed 7 Feb. 2015.

Table 2. Number of U.S. dental schools that were sent survey and responded, by total DDS/DMD enrollment

\begin{tabular}{lcc} 
Enrollment & $\begin{array}{c}\text { Number Sent } \\
\text { Survey }\end{array}$ & $\begin{array}{c}\text { Number That } \\
\text { Responded }\end{array}$ \\
\hline $100-199$ & 7 & 6 \\
$200-299$ & 16 & 7 \\
$300-399$ & 16 & 16 \\
$400-499$ & 14 & 12 \\
500 and above & 11 & 9 \\
Total & 61 & 50
\end{tabular}

Source of enrollment range for schools sent survey: American Dental Association. Survey of dental education: report 1, 2013-14. Table 14. At: www.ada.org/ /media/ADA/Science $\% 20$ and $\% 20$ Research/HPI/Files/SDE1_2013-14\%20_final.ashx. Accessed 7 Feb. 2015.
The emails included an invitation to participate, informed consent elements, and a link to the Qualtrics Academic Integrity Questionnaire. The survey was composed of questions used by McCabe et al. in research that they conducted over the past 25 years, ${ }^{5}$ combined with questions derived from our experiences with dental student cheating. Some additional questions were suggested by public media accounts of dental school cheating incidents in 2006-07. The survey did not request identifying information for individual dental schools or the respondents, thus ensuring their anonymity. The survey consisted of 30 items, with some sub-items to be answered depending on responses to the main items.

The frequencies of responses to survey questions were compiled and analyzed using the Qualtrics evaluation software (Qualtrics, LLC, Provo, UT, USA). Using the Qualtrics software, we conducted statistical analyses on the correlation between the number of reported violations and the measures used by the responding schools (using aggregate data because individual schools were not identified to ensure their anonymity).

\section{Results}

Surveys were completed by the academic deans of 50 U.S dental schools, for a response rate of $82 \%$. The responding dental schools were representative of U.S. dental education institutions according to type (Table 1) and enrollment (Table 2). Responses to the survey questions documented the extensive actions taken by U.S. dental schools to create an environment of academic integrity in their institutions. However, the responses also revealed opportunities for enhancements in policies and actions that could be implemented to address dental student cheating.

Of the total respondents, 29 (58\%) reported that their schools attempted to assess the academic integrity of applicants to their DDS/DMD programs. The most frequent methods employed were interview questions ( 20 schools) and case scenarios (14 schools). Other methods mentioned were essays written by applicants on academic integrity subjects and reviewing applicants' responses to the American Dental Education Association Associated American Dental Schools Application Service (ADEA AADSAS) questions about violation of undergraduate university/college policies or rules governing academic or other forms of misconduct. Schools also scrutinized the content of reference 
letters and required disciplinary check forms to be completed by applicants' undergraduate institutions. Some schools conducted formal background checks on accepted applicants.

Almost all of the respondents (98\%) reported that their schools had policy statements that addressed student academic integrity. Of these policy statements, 90\% included examples of student cheating behaviors, $87 \%$ included expected student academic integrity behaviors, but only $59 \%$ included statements addressing expected faculty academic integrity behaviors. Students were involved in creating the policy statements in $49 \%$ of the schools. Students were involved in the enforcement of academic integrity policies in $80 \%$ of these schools, primarily through service, along with faculty, on hearing/review committees, panels, or councils. Some schools had review panels comprised of only student members.

Among the responding schools, $92 \%$ had an Honor Code governing academic integrity. The code required students to report cheating in $31(69 \%)$ of these schools, with students subject to sanctions for failing to report observed cheating in $22(49 \%)$ of them. In $76 \%$ of the responding schools, students signed a statement attesting to their compliance with the academic integrity policy. The frequency of this attestation varied, from one signing upon matriculation into the program to students signing a statement at each examination/test event.

When asked if their schools provided orientation to the academic integrity policies, $96 \%$ reported providing it for students, but only $26(53 \%)$ provided it for faculty. A variety of institutional rituals were used to emphasize expected student behaviors with respect to academic integrity: $94 \%$ of respondents had a white coat ceremony and $96 \%$ included academic integrity expectations in first-year student orientation activities but only $47 \%$ did so in annual orientation activities for students continuing in the program. Other activities included an Honor Code student signing ceremony, professional ethics programs and curriculum content, a student ethics/professionalism oath-taking ceremony, students' composing a code of conduct for their class, and presentations to students by academic integrity enforcement panel members.

Final examinations ( $91 \%$ of respondents) and tests $(93 \%)$ were almost always proctored, and $80 \%$ stated that their school's security measures to prevent student cheating during tests/examinations were adequate. However, only $33 \%$ of the respondents had a policy on student access to previously administered written examinations/tests, and most of those schools allowed their faculty to choose when/ whether to release previously used examination/test questions. Two schools required the faculty to create new examinations each year and release previously used questions. Two other schools encouraged but did not require these faculty actions.

Although faculty members were required to report suspected cheating in $76 \%$ of the responding schools, $27 \%$ reported feeling that their faculty did so only "rarely." The most common barriers to faculty reporting were reported to be faculty members' reluctance to get involved in the disciplinary process, wariness of student litigation, and belief that the consequences of the disciplinary process would not warrant the "hassle of reporting." Faculty members were also reported to sometimes be unsure of whether they had actually witnessed cheating and to sometimes prefer to address cheating with offending students themselves.

The outcomes of student academic integrity violation disciplinary hearings were disseminated (without identifying information) by $40 \%$ of the responding schools, most often communicated to both students and faculty in the school. Where schools had decided against dissemination of those outcomes, confidentiality and student privacy concerns as well as the Family Educational Rights and Privacy Act (FERPA) were cited as rationales for the decision.

The survey respondents were also asked to name measures to address academic integrity. Among the respondents, 74\% used computer-based student testing, and they were nearly equally divided on whether it had decreased or not changed student cheating. The respondents offered a variety of measures that they believed had improved the academic integrity culture in their schools. Some of these given in free responses to the survey were "following all reported incidents to conclusion and consequence," "raising awareness of faculty and students," "distribution of outcomes of the Peer Review Board Hearings," "enforcing zero tolerance for violations," "shut down all outside computer access during online exams," "use of plagiarism detection software," "cheating case studies in first-year student orientation and ethics and professionalism course," and "student-driven creation of our chapter of the Student Professionalism and Ethics Association."

The respondents listed in free responses a number of measures that they would like to see implemented at their schools. These included "faculty who are willing to report their suspicions," "move away 
from multiple-choice exams to essay, open book, or oral exams," "making outcomes of disciplinary actions known to our school community," "better testing environments," and "adoption of computer testing with randomized question order versions of the same exam." The barriers they named to implementing these measures included "lack of faculty consensus on reuse of exam questions and release of previously used questions" and "students and faculty simply don't want to get involved."

One question on the survey asked: "In your dental school during 2013, how many students were proven to have engaged in each of these academic integrity violations?" A list of cheating violations followed the question. Of the 50 survey respondents, 36 answered this question. The reason for this lower response rate is unclear although the question did not offer a "no incident" response option. Thus, some or all of the nonrespondents may have intended to indicate by their lack of response that there were no

Table 3. Respondents to violations questions by type of school

\begin{tabular}{lcc} 
Type & $\begin{array}{c}\text { Number Sent } \\
\text { Survey }\end{array}$ & $\begin{array}{c}\text { Number Responding } \\
\text { to Violations Questions }\end{array}$ \\
\hline Public & 38 & 22 \\
Private, nonprofit & 20 & 11 \\
Private, state-related & 3 & 3 \\
Total & 61 & $36(59 \%)$
\end{tabular}

reported cheating incidents in their schools. However, four of the 36 respondents did indicate zero incidents in their responses, and one other respondent typed a verbal response of none. The lack of a "no incident" option in the survey question thus did not preclude those respondents from indicating their schools had no reported cheating incidents during 2013. Alternatively, some or all of the 14 nonrespondents purposefully chose not to answer the question. Nonetheless, Table 3 shows the extent to which the 36 responding schools were representative by type of the 61 schools that were sent the survey.

In these 36 responding schools, 141 students were reported to have cheated during 2013 (Table 4), an average of nearly four students per school. Five violations-plagiarism, collaborating on a written assignment when collaboration was forbidden, submitting a written assignment that had been written by another student, copying from another student during a written test/examination, and obtaining test/examination questions before taking the test/ examination-made up $71 \%$ of the 141 reported student violations.

Another survey question asked: "In your dental school, what sanctions/penalties have been applied to students who have been proven to have cheated?" Out of the 50 survey respondents, 46 answered the question. Failure of a course was the most frequently applied sanction, followed in descending frequency by repetition of the course or assignment/test/exami-

Table 4. Total number of students reported for each form of academic integrity violations (36 schools responded to these questions)

Violation

Number of Students

Engaged in plagiarism in writing an assignment for grade credit 26

Collaborated with other students on an assignment for grade credit when such collaboration was forbidden 22

by course instructor

Submitted a written assignment for grade credit that had been written entirely or in part by another student(s) 19

Copied from another student's test/examination during a written test/examination 17

Obtained test/examination questions before taking test/examination 17

Used security code for off-site access to an in-class online test 10

Obtained answers to test/examination questions before taking test/examination 9

Brought "crib notes" or other aids into a written test/examination

Falsified records to obtain credit for patient treatment that was not provided

Falsified faculty approval of preclinical simulation projects

Stole

Falsified faculty approval for patient treatment procedures

Removed tooth from typodont

Lied about clinical experience in written reflection

Changed answers on test review

Gave answers to another student/s during a test/examination for grade credit 
nation, rehabilitative/remediation activities, course grade reduction, and expulsion (Table 5). Repetition of a program year, permanent entry on the transcript, and mention of the violation in reference letters were less frequently used. Written reprimands and suspension for one or more terms were additional penalties mentioned in the free responses. When asked about their comparative perceptions of cheating over time, $57 \%$ of the respondents reported that students in their schools were cheating the same amount as ten years ago, $25 \%$ that students were cheating more today than a decade ago, and $18 \%$ that students were cheating less.

Finally, we analyzed the correlations between the number of reported violations and the measures used by the responding schools. There were no statistically significant correlations.

\section{Discussion}

When asked about assessing the integrity of applicants to their schools, 21 (42\%) of the survey respondents reported that their admission selection process did not include ways attempting to measure the academic integrity of applicants. A few of these respondents noted thinking that measures of academic integrity beyond the factual ADEAAADSAS questions lacked validity and reliability. Analysis of survey responses found no correlation between the use of admission selection measures of applicant academic integrity and the number of proven student cheating incidents in 2013. However, the seriousness of student cheating as a dental education issue seems to justify efforts by schools to attempt to measure applicants' academic integrity.

\section{Academic Integrity Policies and Honor Codes}

While virtually all schools reported having academic integrity policies, $42 \%$ included no expectations concerning the academic integrity behavior of the faculty in the document. Moreover, $47 \%$ of the respondents said their schools did not provide orientation for faculty members to the institution's academic integrity policies. It is perhaps not surprising then that $27 \%$ of respondents reported that faculty members report student cheating only "rarely." Faculty responses to the survey conducted by Andrews et al. led those authors to recommend that schools discuss their academic integrity policies with faculty. ${ }^{2}$ It would seem that faculty members would benefit from frequent orientation to their school's academic integrity policies and the inclusion of explicitly defined expectations for their behavior. Heightened faculty understanding of policies might encourage them to report cheating.

In our survey, $92 \%$ of the 50 responding schools reported having Honor Codes. McCabe et al. advocated the use of Honor Codes to reduce undergraduate college student cheating and reported similar effects on postgraduate students. ${ }^{5}$ Turner and Beemsterboer delineated the structural elements of an effective and valid Honor Code and discussed its potential to enhance the academic integrity environment of dental schools. ${ }^{6}$ Most schools in our study reported that they have students sign a statement attesting to their commitment to abide by the Honor Code: some have students sign once each academic year, but others have them sign at each test/examination. Ariely reported studies demonstrating that having college students read an aspirational statement immediately

Table 5. Sanctions/penalties used for cheating, by number of responding dental schools $(\mathbf{n}=36)$

\begin{tabular}{lc} 
Sanction/Penalty & Number \\
\hline Failure of the course & 36 \\
Repetition of the course & 26 \\
Repetition of the assignment/test/exam & 21 \\
Rehabilitative/remediation activities & 19 \\
Reduction in course grade & 17 \\
Expulsion from program & 17 \\
Other, e.g., documentation in academic file, written reprimand (not in permanent record), suspension for & 16 \\
$\quad$ one or more academic terms & 11 \\
Repetition of program year & 10 \\
Permanent entry on academic record/transcript & 9
\end{tabular}


before an opportunity for potential cheating significantly reduces cheating. ${ }^{7}$ One dental school in our study is operationalizing those findings by having their students sign the following statement whenever they submit an assignment or take a test/examination for grade credit: "On my honor, I attest that I have neither given nor received unauthorized aid in doing this assignment/taking this test/examination." This measure seems to offer potential for reducing cheating in dental schools.

However, Turner and Beemsterboer referred to an Honor Code's requirement for students to report cheating as a "non-toleration element" and discussed the resultant ethical dilemma for students. ${ }^{6}$ They noted that a non-toleration requirement pits students' need to be loyal to their friendship with student colleagues and to belong to their peer group against adherence to the Honor Code. They suggested that a student might privately confront a colleague whom they suspect of cheating, only reporting him or her if the cheating behavior continues. Our survey unfortunately did not ask the extent to which students in the responding schools reported cheating. Future research should address this issue.

While Honor Codes encourage students to aspire to behavior that demonstrates academic integrity, the professional ethics curriculum for dental students offers an opportunity to provide the broadest possible context for this behavior. One respondent to our survey stated that this individual's school had enhanced the academic integrity environment by the addition of cheating case scenarios to first-year student orientation content and the professional ethics course. Most dental schools use a case-based approach to ethics education. Early, repeated, and structured case-based dialogue about the professional ethics implications of cheating would emphasize to students that cheating in dental school potentially affects their future professional competence and, by extension, the welfare of their patients. As one example, Bebeau wrote "The Janet Landry Case," which is available for download on the Center for the Study of Ethical Development website. ${ }^{8}$

\section{Assessment Methods for Dental Students}

McCabe et al. documented over the past 25 years that undergraduate students cheat at an alarming rate. ${ }^{5}$ The challenge for dental educators is to explain for our students the reasons why a dental student cannot continue to cheat like an undergradu- ate student. However, this message will never resonate with dental students unless the dental school environment is significantly different from that of undergraduate education.

To begin this process, dental students must be treated with respect as legitimate professional colleagues by their faculty members. ${ }^{9}$ Students will be more likely to respond positively to expectations for ethical behavior when they are actually treated like professionals. While the dental education experience for our students must be respectful and humanistic, it must also be relevant to contemporary professional practice, in both its pedagogy and assessment methods. Assessment that merely tests student recall of lower learning level "factoids" is all too reminiscent for dental students of the undergraduate college experience. The dental student is more likely to respond to such tests as obstacles to be overcome and, in the extreme reaction, as a barrier in a system to be gamed. When Millennial Generation dental students perceive a test as irrelevant to being a dentist, they are more likely to rationalize cheating as an acceptable means to the end of "getting on to what really matters." Price has observed that Millennials in particular need to see curricular content as relevant because they experience education as representing entry to a career path. ${ }^{10}$ When the assessment is clearly and unquestionably linked to patient care, there is no room for students to ignore the moral imperative of preparing themselves to provide the best patient care they can.

Accordingly, we suggest that dental schools implement clinically realistic student evaluation techniques such as portfolios. Gadbury-Amyot et al. found that reflective portfolios can be used to 1) engage learners in their learning, 2) integrate the behavioral, biomedical, and clinical knowledge and skills, 3) help learners evaluate their own performance, deliberately hypothesizing alternatives and what the expected outcomes of the alternatives might be, and 4) help students self-identify knowledge and experience gaps in their learning. ${ }^{11}$ Such an approach, especially when screened by plagiarism-detecting software, may accomplish increased learning with a concomitant decrease in cheating.

The triple jump examination is another example of authentic evaluation, and alternative approaches to its design should be considered to reduce the number of faculty examiners required..$^{12}$ Schools already have all the materials needed for triple jump case construction in their clinical electronic health records, including patient information, photographs, digitized models, and radiographs. These examinations can 
measure a student's clinical, behavioral, biomedical, and patient communication competence, problemsolving ability, and clinical judgment. Student identity can even be blinded for unbiased evaluation when anonymous examination responses are submitted electronically for analysis by faculty examiners.

Dental schools already widely use the objective structured clinical examination (OSCE) assessment and standardized patients for clinical simulation examinations. ${ }^{13}$ These methods offer the opportunity to assess student knowledge and skill by selectively sampling learning outcomes in simulated clinical settings. Live, structured student case presentations provide another assessment method that is specific to each student. ${ }^{14}$ When student presentations are made to a student/faculty group, peer and faculty evaluation of content, process, and response to questions also contributes to student group learning. One patient experience for one student is thereby expanded to an additional patient experience for many students. In addition, student preparation and presentation of Critically Appraised Topics (CATs) employing evidence-based dentistry Patient/Population/ Problem-Intervention-Comparison-Outcome (PICO) literature searching offers another assessment method that is directly representative of evidence-based dental practice. ${ }^{15}$

As the dental profession becomes more dependent on professional collaboration, more of our pedagogy is being designed to encourage collaborative learning. Problem-based learning, case-based learning, and other small-group learning models are growing in use. If we believe that there is value in collaborative learning, then it should follow that collaborative assessment that evaluates group function and group decision making ought to be employed, particularly in the context of interprofessional health education. Any and all of these methods provide unmistakably clinically relevant assessments that students cannot easily rationalize as being inconsequential to their future career; these methods have the added benefit of being highly resistant to student cheating.

\section{Other School Policies and Activities}

In our study, $96 \%$ of the respondents reported their schools provide first-year student orientation to academic integrity policies, but only $47 \%$ said they repeat the orientation for students continuing in the program. Annual reinforcement of the policies may be desirable. A number of schools reported using presentations to first-year and continuing students by academic integrity enforcement committee members about the cheating cases they have considered and the penalties applied.

Student responses to the Andrews et al. survey were critical of faculty members who used the same examination questions each year and of the lack of consistent, schoolwide policies on the distribution of old exams. ${ }^{2}$ When questions are annually reused by faculty and previously used examinations are circulated without faculty authorization, students who possess or have access to old exams have an unfair advantage over their colleagues who have not seen the previous versions. Student responses to the Andrews et al. survey suggested that instructors should write new questions each year and that there should be consistent schoolwide policies on the distribution of previously used examinations. However, writing effective multiple-choice questions is challenging and time-consuming for already time-pressed faculty members. As an alternative to annually writing new questions, we suggest the use of examination-authoring software that enhances the security of multiple-choice examinations. For a reasonable financial investment, schools can utilize software programs that not only scramble the order of questions, but can alter the order of possible answers. Some forms of software (e.g., ExamSoft, www.learn. examsoft.com) can accommodate the ordering of lists and matching style questions.

Our survey found that only $33 \%$ of the responding schools have a written policy on student access to previously used tests/examinations. Without clear policies on this issue, the risk of cheating incidents based on unevenly available or unauthorized distribution of test questions is significant. Moreover, when students perceive that the assessment environment is unfair to them in any way, their tendency to rationalize cheating as acceptable in their minds may increase. Some schools in our study reported that their increased use of OSCEs, case portfolios, and other non-multiple-choice assessment methods had made the implementation of test item retirement and old exam distribution policies more feasible. Other schools reported using computer-based examinations in which test items can be randomly reordered to produce multiple versions of the same examination that can then be administered to students sitting in close proximity to each other. Dental schools and their faculty members must find definitive solutions to the problem of uneven student access to repeatedly 
used test questions to ensure that inconsistent policies do not contribute to student cheating.

Andrews et al. also reported that faculty members suggested broad dissemination of the outcomes of the enforcement penalties to the school community. ${ }^{2}$ Only $40 \%$ of the schools that responded to our survey reported disseminating this information. The other $60 \%$ cited concerns about maintaining student privacy and complying with FERPA requirements as barriers. However, the schools that do disseminate stated they employ de-identification, redacting, consolidation of details from several incidents, and verbal presentations (instead of written communications) to address privacy concerns. Respondents to the Andrews et al. survey suggested that when the outcomes of reported cheating incidents are not disseminated, students and faculty can come to believe that "nothing ever happens with students who cheat" and faculty reluctance to report cheating is reinforced. ${ }^{2}$ Moreover, students can come to believe that their colleagues who cheat with apparent impunity have an unfair competitive advantage, and this belief can lead them to rationalize cheating "because everyone else is getting away with it."

The incidence of reported cheating in our 2013 survey had increased by almost threefold over that reported for 1998 by Beemsterboer et al. ${ }^{4}$ Unauthorized collaboration among students was the second most common cheating violation reported in our survey. Ironically, dental educators encourage students to collaborate with each other in order to prepare them to function effectively as a health care team member. When the same faculty members forbid students to collaborate on unsupervised takehome assignments, this admonition conflicts with the pervasive encouragement to collaborate. We recommend that faculty members who want to test the independent knowledge of their students should do so in a controlled environment (i.e., not an outof-class, take-home setting). This simple precaution would have prevented $16 \%$ of the reported cheating incidents we found in 2013.

Plagiarism was the most frequently reported type of cheating in 2013 (18\% of incidents). Our survey's question about the types of reported cheating separated the submission of another student's work from other forms of plagiarism. This distinction was made because submitting a student colleague's work for grade credit is intentional unethical behavior, often on the part of two (or more) colluding students. Academic affairs administrators who have dealt with plagiarism frequently find that dental students are often unaware of or do not understand correct paraphrasing technique or even less subtle aspects of plagiarism. Roig found that $30 \%$ of the college professors in his study directly copied some words from a paragraph when they were asked to paraphrase it. ${ }^{16}$ We suggest that schools present curricular content to first-year dental students on avoiding plagiarism. This measure would reduce the likelihood that reported plagiarism is inadvertent and would facilitate the enforcement process.

This study documented that plagiarism was the most frequent cheating violation reported in responding dental schools in 2013. Future research should be conducted to examine the origins and relevance of plagiarism in dental education in the context of our Internet society, where information is freely accessible yet often lacking in attribution of source. Future studies should also attempt to determine whether some dental schools have no reported cheating and whether this is reflective of an institutional environment that perfectly promotes academic honesty or, rather, discourages the reporting of academic dishonesty.

\section{Conclusion}

Responses to our survey, especially to questions asking what measures respondents believed had improved academic integrity in their schools and what measures they want to see implemented in the future, provide us with the well-informed opinions of the academic administrators who routinely deal with reported dental student cheating. Their collective judgment and our own beliefs, based on more than 40 years of combined administrative experience with dental student cheating, lead us to the following recommendations for U.S. dental schools to follow in their DDS/DMD programs:

1. Use admission selection methods to attempt to assess the academic integrity of applicants.

2. Clearly establish cheating within the context of professional ethics by adding cheating case scenarios early in professional ethics courses.

3. Frequently discuss academic integrity policies with faculty members.

4. Delineate expected faculty behaviors in the school's academic integrity policies document.

5. Annually review academic integrity policies with students who are entering and continuing in the program. 
6. Have students sign a statement attesting to their compliance with the Honor Code/academic integrity policies at every testing activity that leads to a grade.

7. Employ examination-authoring software programs to generate new multiple-choice items and different versions of the same multiple-choice tests.

8. Consistently distribute retired test/examination items to all students in the school.

9. Utilize assessment methods that are directly relevant to clinical professional practice.

10. Disseminate outcomes of cheating enforcement actions, using compilation of multiple incidents, redaction, de-identification, and other measures to comply with FERPA and student privacy policies.

11. Avoid take-home exams when assessing independent student knowledge.

12. Add curricular content on avoiding inadvertent plagiarism.

Future studies should be conducted to determine the extent to which these suggestions are implemented in the future by U.S. dental schools and the resultant effects, if any, on reported student cheating.

\section{REFERENCES}

1. Fox K. Asking the big questions: symposium on ethics in education. ADA News 2007;38(12):1,12.

2. Andrews KG, Smith LA, Henzi D, Demps E. Faculty and student perceptions of academic integrity at U.S. and Canadian dental schools. J Dent Educ 2007;71(8):1027-39.

3. American Dental Association. Survey of dental education, report 1, 2013-14. Table 14. At: www.ada.org/ / media/ADA/Science\%20and\%20Research/HPI/Files/ SDE1 2013-14\%20 final.ashx. Accessed 7 Feb. 2015.
4. Beemsterboer PL, Odom JG, Pate TD, Haden NK. Issues of academic integrity in U.S. dental schools. J Dent Educ 2000;64(12):833-8.

5. McCabe DL, Butterfield KD, Trevino LK. Cheating in college: why students do it and what educators can do about it. Baltimore: John Hopkins University Press, 2012.

6. Turner SP, Beemsterboer PL. Enhancing academic integrity: formulating effective honor codes. J Dent Educ 2003;67(10):1122-9.

7. Ariely D. Predictably irrational. Rev. ed. New York: Harper Perennial, 2010.

8. Center for the Study of Ethical Development. 2007. At: www.ethicaldevelopment.ua.edu/BebeauMaterials. Accessed 23 June 2015.

9. Morton NA. Benefits of a humanistic education: a student perspective. J Dent Educ 2008;72(1):45-7.

10. Price C. "Why don't my students think I'm groovy?": the new Rs for engaging millennial learners. Essays Excell Teach 2010;9:29-34.

11. Gadbury-Amyot CC, McCracken MS, Woldt JL, Brennan R. Implementation of portfolio assessment of student competence in two dental school populations. J Dent Educ 2012;76(12):1559-71.

12. Navazesh M, Rich SK, Chopiuk NB, Keim RG. Triple jump examinations for dental student assessment. J Dent Educ 2013;77(10):1315-20.

13. Graham R, Bitzer LAZ. Reliability and predictive validity of a comprehensive preclinical OSCE in dental education. J Dent Educ 2013;77(2):161-7.

14. Townsend JA. Dental rounds: an evolving process of curriculum integration at the LSU School of Dentistry. J Dent Educ 2014;78(5):796-802.

15. Hendricson WD. Changes in educational methodologies in predoctoral dental education: finding the perfect intersection. J Dent Educ 2012;76:118-41.

16. Roig M. Plagiarism and paraphrasing criteria of college and university professors. Ethics Behav 2001;11:307-23. 